\title{
Diagnosis of Primary Cutaneous Amyloidosis by Rapid 4,6-Diamidino-2-Phenylindole Staining
}

\author{
Junchen Chen Huan Yang Zhijun Xu Ping Lu Liyan Yuan Yaohua Xue \\ Ruzeng Xue Bin Yang
}

Department of Dermatology, Dermatology Hospital, Southern Medical University, Guangzhou, China

\section{Keywords}

4,6-Diamidino-2-phenylindole $\cdot$ Congo red $\cdot$ Hematoxylineosin $\cdot$ Keratin $\cdot$ Primary cutaneous amyloidosis

\begin{abstract}
Background: Quick and accurate diagnosis of primary cutaneous amyloidosis (PCA) may be difficult because its symptoms are often subtle and nonspecific. Objective: We sought to review the literature on the roles of various staining methods in the diagnosis of amyloidosis and demonstrate added benefits of using rapid 4,6-diamidino-2-phenylindole (DAPI) staining in the diagnosis of PCA. Methods: Three groups of cases, namely, PCA, neurodermatitis, and prurigo nodularis, were retrieved from a computerized pathology database for study, and their paraffin-embedded tissue blocks were cut following standard procedures. The tissue sections were stained with three stains: hematoxylin-eosin $(\mathrm{HE})$, Congo red, and DAPI stains, and examined under the microscope to compare the staining patterns of these three methods. We also performed amyloid keratin and apolipoprotein $E$ (APOE) staining on the sections of PCA in order to further support our conclusion. The PCA sections were read by junior and senior dermatopathologists for comparison. Results: The sensitivity of DAPI staining for PCA was significantly higher than that of Congo red staining and HE staining $(p<0.001)$. This statement holds true whether the ex-
\end{abstract}

karger@karger.com www.karger.com/drm

Karger $\stackrel{\text { ' }}{5}$

GOPEN ACCESS
C 2021 The Author(s).

Published by S. Karger AG, Basel

This is an Open Access article licensed under the Creative Commons Attribution-NonCommercial-4.0 International License (CC BY-NC) (http://www.karger.com/Services/OpenAccessLicense), applicable to the online version of the article only. Usage and distribution for commercial purposes requires written permission. periment was grouped in one sample or was divided into groups of junior and senior dermatopathologists $(p<0.001)$. The DAPI-positive staining areas, except for the nuclei, were consistent with the amyloid deposition areas. In this study, DAPI staining had a sensitivity of $98.6 \%$ and a specificity of $100 \%$. Conclusion: DAPI staining could serve as a useful technique to establish the diagnosis of PCA, and its high efficacy in diagnosing PCA makes it less dependent on the experience levels of the evaluators. Additionally, the binding of DAPI to the A-T-rich sequence of double-stranded DNA suggests that amyloid may contain DNA or a similarly structured nucleic acid.

(C) 2021 The Author(s).

Published by S. Karger AG, Basel

\section{Introduction}

Primary cutaneous amyloidosis (PCA) is a chronic dermatosis characterized by amyloid deposition in the dermis without involvement of internal organs. PCA occurs globally but is more common in Southeast Asia [1]. The subtypes of PCA include lichen amyloidosis (LA),

Junchen Chen, Huan Yang, and Zhijun Xu contributed equally to this work and should be considered joint first authors. Ruzeng Xue and Bin Yang contributed equally to this work and should be considered joint corresponding authors.
Correspondence to:

Ruzeng Xue, xueruzeng@163.com Bin Yang, yangbin1@smu.edu.cn 
macular amyloidosis (MA), and nodular amyloidosis (NA) [2]. The combined appearance of lichenoid amyloidosis and MA is described as biphasic amyloidosis [3]. Clinically, PCA presents with multiple localized hyperpigmented macules or grouped papules generally associated with severe pruritus, compromising the quality of life in varying degrees $[4,5]$. Clinical appearance differs between LA and MA: LA most commonly presents as intensely pruritic papules, sometimes coalescent with lichenification, while MA is characterized by flat hyperpigmented macules that may form a reticular or confluent pattern. These two entities cannot be clearly distinguished on the histological ground $[2,6,7]$. In addition, symptoms of PCA are subtle and nonspecific, often mimicking manifestations of lichenoid dermatoses, neurodermatitis, and prurigo nodularis.

Confirming deposits of amyloid requires special techniques, such as special stains, immunohistochemistry (IHC), immunofluorescence technique. PCA can be recognized on the hematoxylin-eosin (HE)-stained sections by the presence of varyingly sized, oval-shaped, or agglomerate, homogeneously eosinophilic material in the dermal papillae. Increased melanin in the basal layer, vacuolar change of basal keratinocytes, and slight acanthosis are also helpful diagnostic clues $[6,8]$. However, the amyloid deposits can be subtle on HE-stained sections when the amount of amyloid is small so that its diagnosis is missed by unary pathologists. More importantly, amyloid could be confused with hyaline degeneration with similar homogenized, eosinophilic appearances histologically. Therefore, special staining technology has become an indispensable adjunct for the diagnosis of PCA.

Congo red dye was created by Bottiger in 1884 and its characteristic binding to amyloid was discovered by Bennhold in $1922[9,10]$. Bennhold and Divry also demonstrated that yellow-green birefringence could be observed under a polarized light when amyloid was stained with Congo red. This feature has been served as the first and main diagnostic criterion for amyloid $[10,11]$. Unfortunately, this method has lost its popularity since 2001 due to low fluorescence intensity and false-positive results [11]. The use of IHC, targeting different chemical types of amyloid protein, was recently recommended to identify and subtype amyloidosis. Yet, IHC is relatively expensive and complicated, and its results can be affected by many factors, such as the preanalytical and analytical variables related to tissue fixation and processing, the equipment and methodology used, the nature of the antibodies, the method of antigen retrieval, and the interpretation of staining results by the pathologist. Therefore, we seek to find a simpler and stabler staining method to diagnose PCA.

4,6-Diamidino-2-phenylindole (DAPI) is a fluorochrome, which can penetrate the cell membrane and bind strongly to the double-stranded DNA in the nuclei, playing a role in labelling through increasing the fluorescence by approximately 20 -fold [12]. There is little doubt that the fluorochrome molecule of DAPI binds to the minor grove of consecutive (3-4 base pairs) A-T-rich sequences of double-stranded DNA, but its binding mechanism is still not fully understood [13]. The possible formation of DAPI binding to nucleic acid includes minor groove binding, intercalation, and condensation [13]. Beccia et al. [14] found that the mode of DAPI/DNA interaction could be changed from groove binding to intercalation under particular conditions. In addition, DAPI could also bind to other sequences of double-stranded DNA or double-stranded RNA. However, fluorescence emitted by these complexes is much lower [12,13].

The DAPI stain is popularly applied in diagnosing renal amyloidosis at present time and can even detect a small amount of amyloid paraprotein [15]. Moreover, Matsuura et al. [15] showed that DAPI can be useful to distinguish immunoglobulin light-chain amyloid type (AL) from the acute-phase reactant serum amyloid A, but the specific mechanism is unknown. In NA, the deposit is of AL type, which is consistent with those reported in the literature, whereas in LA and MA, the protein deposit is of keratin-associated amyloid type (AK) [16-18]. However, the rarity of NA cases makes conducting a statistically significant study very difficult.

The composition of amyloid is complex, consisting of amyloid fibrillin and other components including serum amyloid P [19], APOE [20, 21]. In the past, we had performed triple staining of DAPI, keratin, and APOE immunofluorescence on each of the established PCA cases that are in different clinical stages. Our goal was to classify the components of amyloid deposits in Chinese patients and seek to understand the pathogenesis of PCA. We found that, within the same case, the DAPI-positive areas were concentrated in the dermal papilla, coinciding with the areas that stain positive for keratin and APOE. Our observation corroborates those reported in the literature $[15,21,22]$. In this study, we explored the feasibility of the application of DAPI in the diagnosis of LA and MA.

Neurodermatitis and prurigo nodularis are common pruritic skin conditions with clinical manifestations that are similar to those of PCA. The collagen degeneration occurring in these two diseases may closely mimic amyloid histologically. Also, the histological appearance of the de- 
Fig. 1. Flowchart of Materials and Meth-

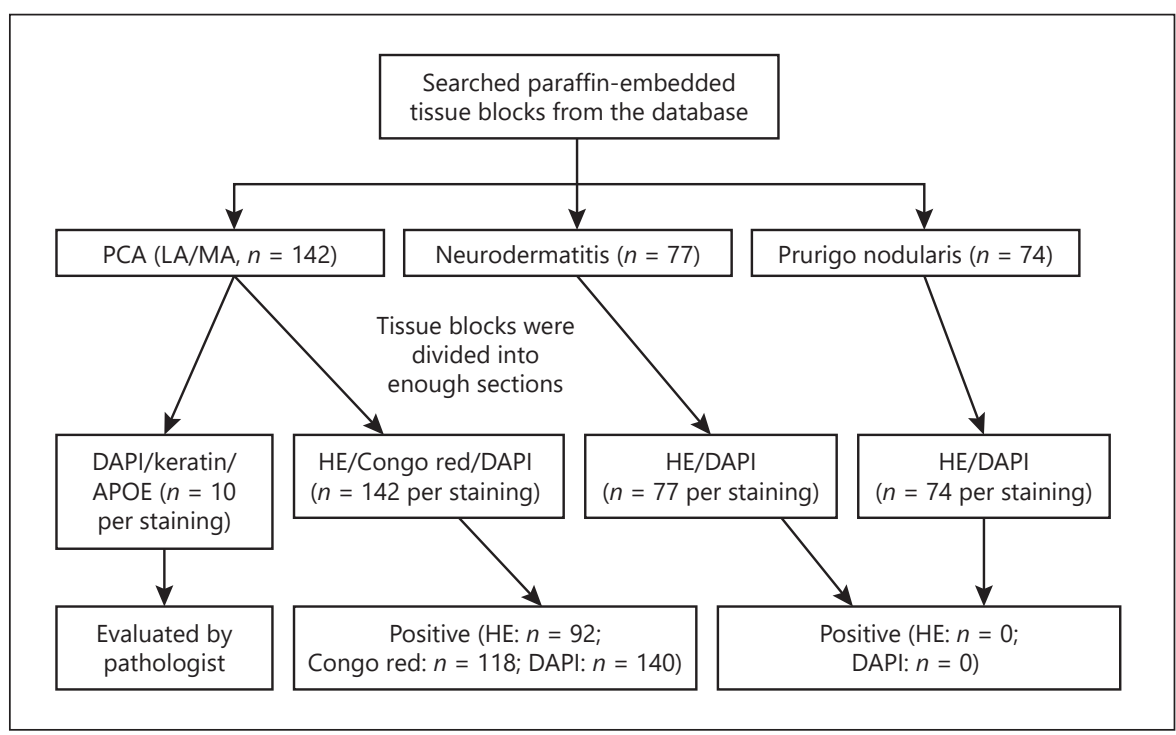
ods.

posits observed in lipoid proteinosis and porphyria may simulate amyloid [23]. Therefore, the above-mentioned conditions served as negative controls for the DAPI stain in our study. We proved that the result of the DAPI staining in $\mathrm{AK}$ is similar to that reported in literature and its staining effect is significantly better than that of Congo red and HE, suggesting that DAPI staining can serve as a simple and stable method to diagnose PCA $[15,22]$.

\section{Materials and Methods}

We searched three groups of relevant diseases, whose clinical records were retrievable, from a computerized pathology database at the Dermatology Hospital of Southern Medical University between 2017 and 2019. We selected 142 cases with PCA (LA and MA types), 77 cases with neurodermatitis, and 74 cases with prurigo nodularis (Fig. 1). The initial diagnosis of all cases was made by comprehensive consideration of clinical manifestations and pathological results. All paraffin-embedded tissue blocks from the specimens of these patients were retained for study.

Histology

Using standard techniques, $3-\mu \mathrm{m}$ sections from each PCA case were stained with HE for routine histological assessment and with Congo red (Solarbio, G1532) according to Stokes' method [24]. Detection of apple-green birefringence on polarizing microscopy confirmed the presence of amyloid. For cases of neurodermatitis and prurigo nodularis, the sections were stained with $\mathrm{HE}$ for morphology.

\section{Immunofluorescence and Imaging}

DAPI staining of these samples (PCA, neurodermatitis, and prurigo nodularis) only took a short time to complete. The paraffin-embedded sections were dewaxed and rehydrated according to standard procedures; after that, $50 \mu \mathrm{L}$ of DAPI mounting medium (stock solution, abcam, \#ab104139) was applied on top of the spec- imen and spread out evenly by tilting sections back and forth or stirring the medium with a $0.2-\mathrm{mL}$ plastic pipette tip without touching the sections. The incubation was left standing at room temperature for about $5 \mathrm{~min}$. A cover slip was applied and then sealed with nail polish. To perform keratin or APOE immunofluorescence staining for sections of PCA, paraffin sections were dewaxed and rehydrated, and boiled antigen retrieval solution with a $\mathrm{pH}$ of 9.0 was used to perform antigen retrieval. Sections were then incubated with mouse monoclonal anti-cytokeratin (1:40; GeneTex, \#34ßE12), goat anti-apolipoprotein E (1:200; abcam, \#ab32897). To perform DAPI-keratin-APOE triple staining, donkey anti-mouse IgG Alexa Fluor 488 (1:500; Invitrogen, \#A21202) and donkey anti-goat IgG Alexa Fluor plus 647 (1:500; Invitrogen, \#A32849) were used as secondary fluorescence antibody for the sections above. The sections were then counterstained with DAPI (abcam, \#ab104139), and cover slips were applied to cover the sections carefully avoiding air bubbles. Finally, the specimens were ready for visualization and images were acquired using a confocal microscope (Nikon A1+).

The senior dermatopathologists with over 5 years of working experience interpreted the staining results of both PCA cases and cases serving as negative controls, and these interpretations were taken as the gold standards, whereas the junior dermatopathologists with less than 1 year of working experience interpreted only the staining results of PCA for comparison. Our experiments were performed blindly: dermatopathologists reviewed each case without knowing the specific diagnosis rendered for that case.

\section{Evaluation of Special Stain Sections}

HE-stained sections were evaluated for amorphous eosinophilic material in the dermis. Congo red-stained sections were evaluated for amorphous brick-red material inside the dermal papillae under routine microscope and apple-green birefringence under polarizing microscopy in the papillary and upper dermis.

\section{Evaluation of DAPI-Stained Sections}

DAPI-stained sections were evaluated for oval-shaped, cordlike or cloud-like blue material deposits inside the dermal papillae 
Table 1. Difference and positive rate of various staining in PCA

\begin{tabular}{lccccc}
\hline Staining & Positive & Negative & Total & $\chi^{2}$ & $p$ value \\
\hline HE & $92(64.8 \%)$ & $50(35.2 \%)$ & $142(100 \%)$ & & \\
DAPI & $140(98.6 \%)$ & $2(1.4 \%)$ & $142(100 \%)$ & & $<0.001$ \\
Total & $232(81.7 \%)$ & $52(18.3 \%)$ & $284(100 \%)$ & 54.239 & \\
Congo red & $118(83.1 \%)$ & $24(16.9 \%)$ & $142(100 \%)$ & & $<0.001$ \\
DAPI & $140(98.6 \%)$ & $2(1.4 \%)$ & $142(100 \%)$ & & 20.491 \\
Total & $258(90.8 \%)$ & $26(9.2 \%)$ & $284(100 \%)$ & & \\
\hline
\end{tabular}

Table 2. Specificity and sensitivity of DAPI staining

\begin{tabular}{llcl}
\hline & \multicolumn{3}{l}{ Group } \\
\cline { 2 - 4 } DAPI & PCA & Non-PCA & Total \\
\hline Positive & $140(98.6 \%)$ & $0(0 \%)$ & $140(47.8 \%)$ \\
Negative & $2(1.4 \%)$ & $151(100 \%)$ & $153(52.2 \%)$ \\
\hline Total & $142(100 \%)$ & $151(100 \%)$ & $293(100 \%)$ \\
\hline
\end{tabular}

Sensitivity (the probability that a patient with amyloid deposition inside dermal papillae tests positive) is 140/142 or 98.6\%. Specificity (the probability that a patient without amyloid deposition inside dermal papillae tests negative) is $151 / 151$ or $100 \%$. Non-PCA, includes neurodermatitis and nodular pruritus.

under excitation at $450 \mathrm{~nm}$ by confocal microscope (Nikon $\mathrm{A} 1+$ ). In each section, the DAPI staining of epidermal keratinocytes was considered as an internal positive control for DAPI, but the true fluorescence intensity of DAPI in amyloid was relatively low. Sections from lipoid proteinosis and porphyria were considered as negative controls for DAPI staining.

\section{Evaluation of Keratin-Stained Sections and APOE-Stained} Sections

Keratin-stained sections were evaluated for green immunofluorescence in amyloid deposits under excitation at $488 \mathrm{~nm}$ by confocal microscope, which was considered as a positive reaction. The purple immunofluorescence scattered in the dermis under excitation at $647 \mathrm{~nm}$ by confocal microscope was considered to be a positive reaction of APOE staining.

\section{Exclusion Criteria}

The specimens without enough lesional tissue for study were excluded. In negative control cases, those with PCA in clinical or pathological differential diagnosis were excluded [25].

\section{Statistical Analysis}

We conducted a statistical analysis to verify the credibility of the results. Differences in the PCA-positive rate between DAPI staining and the other two staining methods were evaluated by $\chi^{2}$ test. The positive rate of $\mathrm{HE}$, Congo red, and DAPI staining results interpreted by junior and senior dermatopathologists was statistically compared by $\chi^{2}$ test. Statistical analysis was performed in SPSS version 25.0. $p<0.05$ was considered statistically significant.

Diagnosis of Primary Cutaneous

Amyloidosis

\section{Results}

\section{Clinical and Statistical Data}

The results of total 142 cases of PCA are shown in Table 1 . The positive cases identified by HE, Congo red, and DAPI staining were 92,118 , and 140 , respectively, yielding a sensitivity of $64.8 \%, 83.1 \%$, and $98.6 \%$, respectively. The sensitivity of DAPI staining was significantly higher than that of Congo red and HE staining $(p<0.001)$. Two of the 142 cases with negative DAPI staining results, were regarded as false negative results because the characteristics of PCA were confirmed by HE and Congo red stains. From our study, the specificity was $100 \%$, as shown in Table 2 .

Junior and senior dermatopathologists interpreted blindly the PCA sections using different staining methods. The results shown in Table 3 demonstrate that the sensitivity of the HE staining in detecting amyloid is distinctly higher in the senior group than that of the junior counterpart $(p<0.05)$, but the sensitivity of DAPI staining between the junior and senior dermatopathologists was not different. The sensitivities of HE, Congo red, and DAPI stains in diagnosing amyloidosis, when in the hands of junior dermatopathologists, were $52.1 \%, 71.1 \%$, and $98.6 \%$, respectively; and $64.8 \%, 83.1 \%$, and $98.6 \%$, respectively, as determined by senior dermatopathologists. The sensitivity of the DAPI group was significantly higher than that of the Congo red and HE groups $(p<$ 0.001 in both comparisons). Moreover, no cases of nodular prurigo and neurodermatitis were diagnosed as amyloidosis by HE staining and DAPI staining.

\section{Histopathology and Immunofluorescence Performance}

The HE-stained PCA tissues showed a small amount of amorphous eosinophilic material with uniform fissures inside the expanded dermal papillae under microscope. Yet, the surrounding tissue was stained with light red, and the boundary between deposits and surrounding dermal tissue was not clear (Fig. 2a). In addition, the result revealed other characteristics of PCA, such as slight 

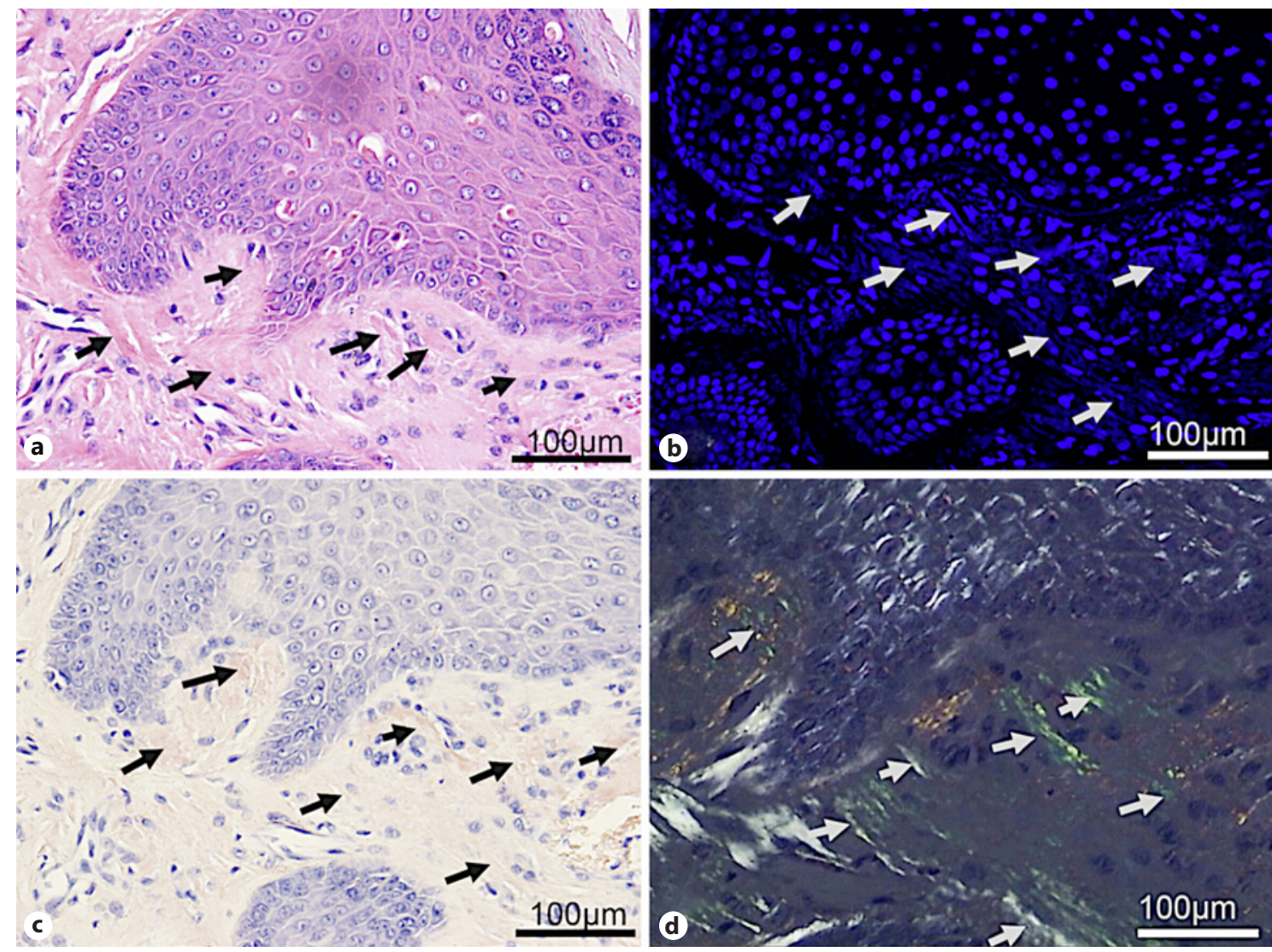

Fig. 2. Microscopic appearance of the tissue with deposition of amyloid. a Amorphous eosinophilic material with uniform fissures inside the expanded dermal papillae and slight hyperkeratosis in the stratum corneum (hematoxylin and eosin staining). b Cloud-like blue uniform clumps of different sizes inside the dermal papillae (DAPI staining). c Cloud-shaped amorphous brick-red material inside the dermal papillae (Congo red staining). d Apple-green birefringence on polarizing microscopy (Congo red staining). Magnification for all panels was $\times 200$.

Table 3. Results of junior and senior dermatopathologists in the diagnosis of PCA after different staining (all values of stain are average of the dermatopathologists in the same group)

\begin{tabular}{|c|c|c|c|c|c|c|c|}
\hline & $\mathrm{HE}$ & Congo red & DAPI & $\begin{array}{l}\chi^{2} \\
\text { (HE/DAPI) }\end{array}$ & $p$ value & $\begin{array}{l}\chi^{2} \\
\text { (Congo red/DAPI) }\end{array}$ & $p$ value \\
\hline \multicolumn{8}{|l|}{ Senior } \\
\hline Positive & $74(52.1 \%)$ & 101 (71.1\%) & $140(98.6 \%)$ & & & & \\
\hline Negative & $68(47.9 \%)$ & $41(28.9 \%)$ & $2(1.4 \%)$ & & & & \\
\hline Total & $142(100 \%)$ & $142(100 \%)$ & $142(100 \%)$ & 82.584 & $<0.001$ & 41.683 & $<0.001$ \\
\hline \multicolumn{8}{|l|}{ Junior } \\
\hline Positive & $92(64.8 \%)$ & 118 (83.1\%) & $140(98.6 \%)$ & & & & \\
\hline Negative & $50(35.2 \%)$ & $24(16.9 \%)$ & $2(1.4 \%)$ & & & & \\
\hline Total & $142(100 \%)$ & $142(100 \%)$ & $142(100 \%)$ & 54.239 & $<0.001$ & 20.491 & $<0.001$ \\
\hline$\chi^{2}$ (junior/senior) & 4.698 & 5.766 & & & & & \\
\hline$p$ value & 0.03 & 0.016 & & & & & \\
\hline
\end{tabular}


Fig. 3. DAPI staining of nodular pruritus and neurodermatitis; no similar cloud-like material was found inside the dermal papilla. a Nodular pruritus. b Neurodermatitis. The white dashed line marks the basal layer (DAPI staining, magnification $\times 200$ ).

Fig. 4. Flocculent deposits without nuclei dyed with DAPI coincided with the deposition of keratin and APOE. a DAPI staining. b Immunofluorescence staining for keratin. c Immunofluorescence staining for APOE. d Triple staining of DAPI (blue), keratin (green), and APOE (purple). Magnification for all panels was $\times 300$.
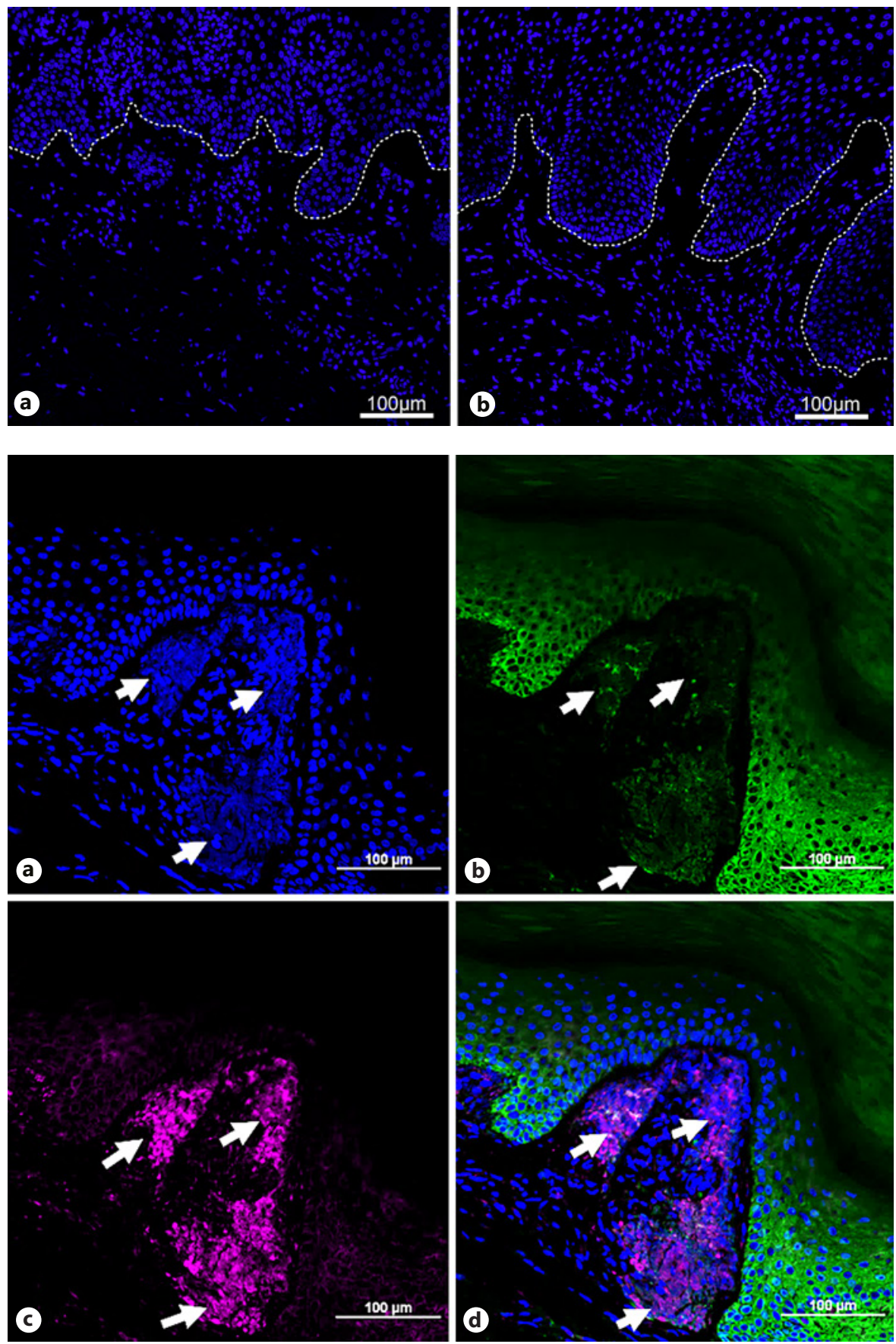

hyperkeratosis and acanthosis. The Congo red-stained PCA showed a cloud-shaped amorphous brick-red material inside the dermal papillae and exhibited apple-green birefringence on polarizing microscopy in the papillary and upper dermis (Fig. 2c, d). The results were analogous to those reported in literature [7].

DAPI staining revealed that amyloid had oval-shaped or cloud-like blue clumps of different sizes inside the der- mal papillae, which were clearly separated from the surrounding epidermis and dermal tissue (Fig. 2b). The DAPI-positive areas overlapped with that of the Congo red stain to a great extent. This result initially indicated that the DAPI-positive staining areas, except for the nuclei, were consistent with the areas of amyloid deposition, as reported in the literature $[15,22]$. No similar cloud-like material was found inside the dermal papillae in the DAPI 
staining results of neurodermatitis and nodular pruritus (Fig. 3a, b).

In order to verify whether DAPI accurately recognizes amyloid, we performed DAPI, amyloid K, and APOE staining on 10 patients with PCA. The results are shown in Figure 4. DAPI-positive areas without nuclei presented as cord-like or cloud-like blue material under confocal microscope, which corresponded to the deposition of paraprotein in every patient with $\mathrm{AK}$ amyloidosis (Fig. $4 \mathrm{a}-\mathrm{c}$ ). The DAPI-keratin-APOE triple staining on the same sections of PCA strongly showed the consistency of overlapping staining (Fig. 4d). Moreover, the brightblue DAPI-positive areas without nuclei in the dermal papillae were not observed in the cases of lipoid proteinosis and porphyria (online suppl. Fig. 5a, b, available at www.karger.com/doi/10.1159/000518082).

\section{Discussion}

PCA is a chronic condition that deposits amyloid in previously normal skin without systemic involvement. Although PCA is common clinically, its rapid and accurate diagnosis is often difficult since this disease often needs to be distinguished from chronic eczema, neurodermatitis, and nodular prurigo due to the similar clinical features. PCA have an obvious impact on the quality of life for patients because of symptoms such as intense itching and unsightly skin appearance. Hence, the rapid and accurate diagnosis of PCA is of great importance for its management.

In this study, we have proved that DAPI staining is useful in the diagnosis of PCA (AK type) since almost all the DAPI-positive staining areas of PCA overlapped with the amyloid deposition areas of Congo-red/HE-positive area except for two cases, and this finding was similar to the staining performance of DAPI reported in the literature $[15,22]$. More importantly, the triple staining (DAPI, keratin, and APOE stains) on the same sections of PCA further proved that the DAPI-positive areas corresponded with the deposition of paraprotein in patients with AK amyloidosis. Furthermore, DAPI staining showed no amyloid deposition in nodular prurigo and neurodermatitis. Based on the results, the DAPI staining demonstrated the best sensitivity in diagnosing amyloidosis, which is significantly higher than those of $\mathrm{HE}$ and Congo red stains. The detection of amyloid deposits by DAPI staining in LA and MA types of PCA enables an accurate diagnosis. Unfortunately, currently we could not use NA (AL type) as a comparison due to few cases, but the literature had con- firmed that DAPI could recognize AL [15]. We are in search of more cases of NA to conduct further studies. Nevertheless, we have proved that DAPI can aid in the diagnosis of LA and MA.

Meanwhile, DAPI is a fluorescent stain that labels DNA and our large bodies of research have proved that almost all the amyloid deposited in the dermis of PCA are stained positive for DAPI. The area where DAPI binds to amyloid without the nucleic of the basal layer produces blue fluorescence under excitation at $450 \mathrm{~nm}$ by confocal microscope, which was similar to the observations of DAPI's binding to DNA $[14,22]$. The above evidence supports a novel idea that these amyloids of PCA (LA and MA types) may contain DNA or an unrecognized structure of nucleic acids. Yang et al. [22] had proved that hyperpigmented lesions of patients with amyloidosis cutis dyschromica (ACD) exhibited significantly increased amounts of DNA/keratin-positive amyloid deposits in the papillary dermis, but the DNA signals in amyloid deposits were not as intact as the nuclei inside the cells, suggesting presence of DNA debris in the amyloid deposits. At the same time, ultrastructural evidence had demonstrated cytoplasmic and nuclear fragments in the amyloid deposits. The electron microscopic findings included typical filaments of amyloid of 6-10 nm thickness in the papillary dermis and massive amounts of fibrillar aggregates in the dermalepidermal junction or the cytoplasm of hyperpigmented lesion in the patients with ACD $[22,26]$. Ionescu et al. [7] had also found some dotted or cloud-like agglomerates with high refractive index from the dermoepidermal junction of the affected skin by reflectance confocal microscopy.

However, the specific structure of these nucleic acids and the mechanisms of their formation are not fully clear. PCA is considered to be a multi-factorial disease and genetic factor plays a critical role in the development of PCA. In recent years, there has been evidence that PCA is strongly associated with missense mutations of OSMR (encoding oncostatin M receptor-beta [OSMRb] [MIM: 105250]) and IL31RA (encoding interleukin-31 receptor-alpha [MIM: 613955]) [22, 27-31]. It is worth mentioning that Yang et al. [22] had proved that the lossof-function mutations in glycoprotein nonmetastatic gene B (GPNMB [MIM: 604368]) could cause autosomal-recessive ACD and potentially lead to increased or chronic inflammatory responses, misfolded protein aggregates, reduced clearance of cellular debris and protein aggregates, and keratinocyte degeneration. The last entity was considered to provide potential theoretical basis 
into the etiology of amyloidosis in ACD. In any case, the full component of amyloid and the specific mechanism of its formation in PCA need to be further studied by research.

DAPI staining is as simple and fast as Congo red staining and its evaluation under the microscope is easier than that of Congo red. Literature showed that the sections dyed with classic Congo red staining demonstrate a low contrast between positive and negative areas, making it difficult to distinguish the positive region from the surrounding normal tissue. The difficulty in interpretations may increase the possibility of false positivity $[10,11]$. In contrast, the false positivity of DAPI in our study is zero.

We evaluated the effect of DAPI staining in the diagnosis of PCA by dermatopathologists with varying working experience. We found that the diagnostic accuracy of the senior dermatopathologist was significantly higher than that of the junior dermatopathologist when using HE staining $(p<0.05)$. This finding illustrated that our inquiry is of clinical importance. The diagnostic accuracy of dermatopathologists using DAPI staining was significantly higher than that using HE staining, especially in the junior group $(p<0.001)$. This result supports the notion that DAPI staining can effectively enhance the accuracy of dermatopathologists in diagnosing PCA, and it further enhances the diagnostic accuracy of the novice. Moreover, the time spent in DAPI staining was short, which could greatly improve the turnaround time of the pathology report. However, a certain subjective error existed in this study because of the small number of dermatopathologists involved. Nevertheless, we showed that DAPI can serve as a stable and simple staining method for the diagnosis of PCA.

DAPI staining has been used to diagnose renal amyloidosis, but we believe that no literature has reported the wide application of DAPI staining in PCA diagnosis. In a published study, Matsuura et al. [15] confirmed that DAPI staining was capable of showing amyloid deposition areas in the sections of renal amyloidosis more efficiently compared with Congo red staining. Their results are similar to that of our study and had proven that DAPI staining can be used to detect amyloid deposition [15]. However, their samples are limited; therefore, their results need to be further verified. In contrast, the samples in our study are much larger and our results are statistically significant, making our conclusion more reliable.

Recently, it is recommended to use IHC to identify amyloid. Sari Aslan et al. [25] had proved that amyloid was strongly stained by cytokeratin 5 antibody and high

Diagnosis of Primary Cutaneous

Amyloidosis molecular weight keratin (34betaE12), which can be used to diagnose MA. However, using either cytokeratin 5 antibody or high molecular weight keratin alone does not improve the detection of amyloid compared with HE staining. This type of IHC procedure takes a long time and is complicated due to many factors, such as the variations related to tissue processing, the equipment and methodology used, the nature of the antibodies, and the method of antigen retrieval. Our DAPI staining, on the other hand, is relatively simple and reproducible because of the simple protocol and ready availability of reagents. In recent years, new staining techniques and fluorescent dyes for amyloid have been reported. For example, Gusel'nikova et al. [32] had confirmed that the sensitivity and specificity of DSNAF method for detecting amyloid is equivalent to that of Congo red staining. DSNAF is a fluorene-based analogue of Congo red offering superior staining. We hope that, through collected efforts, an amyloid stain with a better efficacy combining with either a simpler procedure or a cheaper cost will emerge to aid in the diagnosis of PCA and its mechanism research.

In summary, DAPI staining method offers a simple and rapid protocol to accurately detect amyloid deposits for diagnosing lichenoid amyloidosis and MA. Early and accurate diagnosis is necessary to help patients enhance their quality of life. A fluorescent microscope and expertise training are needed to interpret the DAPI-stained sections. These necessities do not add costs for advanced dermatology departments where the fluorescent microscope is readily available. They, however, pose a problem for dermatology departments in remote areas. Nevertheless, we believe that the DAPI stain serves as a useful adjunct for the diagnosis of PCA (LA and MA types) and helps to distinguish PCA from its mimics. This stain may have a potential to provide theoretical support for further exploring the components of amyloid and its formation mechanisms.

\section{Key Message}

DAPI staining is useful for rapid diagnosis of primary cutaneous amyloidosis.

\section{Acknowledgement}

We are grateful to the clinician for revising our paper: Wei Su, MD, Midwest center for dermatology \& cosmetic surgery, Clinton township, Michigan. 


\section{Statement of Ethics}

The paper is exempt from ethical committee approval. We have obtained the consent of Dermatology Hospital of Southern Medical University to use paraffin-embedded tissue blocks, which are legally owned by the hospital. We did not directly collect any materials from the patients and did not infringe on the interests of the patients during this study.

\section{Conflict of Interest Statement}

The authors have declared that no conflict of interest exists.

\section{Funding Sources}

This research was financially supported by The National Natural Science Foundation of China (grant No. 81703123 and 81972924).

\section{Author Contributions}

Z.X. drafted the manuscript. R.X. and B.Y. edited it. J.C., Z.X., and R.X. reviewed and analyzed histopathological sections. Z.X., P.L., and H.Y. performed the statistical analysis. J.C., H.Y., and L.Y. prepared the sections and performed staining. J.C., H.Y., Y.X., R.X., and B.Y. designed the study. All authors read and approved the manuscript.

\section{References}

1 Tan T. Epidemiology of primary cutaneous amyloidoses in southeast Asia. Clin Dermatol. 1990 Apr-Jun;8(2):20-4.

2 Love WE, Miedler JD, Smith MK, Mostow EN, Cooper KD, Gilliam AC. The spectrum of primary cutaneous nodular amyloidosis: Two illustrative cases. J Am Acad Dermatol. 2008 Feb; 58(2 Suppl 1):S33-5.

3 Wang WJ. Clinical features of cutaneous amyloidoses. Clin Dermatol. 1990 Apr-Jun;8(2): 13-9.

4 Weidner T, Illing T, Elsner P. Primary Localized Cutaneous Amyloidosis: A Systematic Treatment Review. Am J Clin Dermatol. 2017 Oct;18(5):629-42.

5 Lu P, Wu FF, Rong ZL, Fang C, Deng CC, Bin $\mathrm{LH}$, et al. Clinical and genetic features of Chinese patients with lichen and macular primary localized cutaneous amyloidosis. Clin Exp Dermatol. 2019 Jun;44(4):e110-7.

6 Groves RW. Amyloidosis. In: Dermatology; Bolognia JL, Schaffer JV, Cerroni L, eds; Elsevier: Amsterdam, The Netherlands, 2017; pp. 754-760. ISBN 978-0702062759.

7 Ionescu A-M, Ilie M-A, Chitu V, Razvan A, Lixandru $\mathrm{D}$, Tanase $\mathrm{C}$, et al. In vivo Diagnosis of Primary Cutaneous Amyloidosis -the Role of Reflectance Confocal Microscopy. Diagnostics. 2019 Jun 27;9(3):66.

8 Schreml S, Szeimies RM, Vogt T, Landthaler M, Schroeder J, Babilas P. Cutaneous amyloidoses and systemic amyloidoses with cutaneous involvement. Eur J Dermatol. 2010 Mar-Apr; 20(2):152-60

9 Frid P, Anisimov SV, Popovic N. Congo red and protein aggregation in neurodegenerative diseases. Brain Res Rev. 2007 Jan;53(1):135-60.

10 Fernandez-Flores A. Cutaneous amyloidosis: a concept review. Am J Dermatopathol. 2012 Feb;34(1):1-17; quiz 15-7.

11 Vowles GH, Francis RJ. Amyloid. 5th ed. In: Bancroft JD, Gamble M, editors. Theory and Practice of Histological Techniques. London: Churchill Livingstone; 2001. p. 303-24.
12 Chazotte B. Labeling nuclear DNA using DAPI. Cold Spring Harb Protoc. 2011 Jan 1; 2011(1):pdb.

13 Kapuscinski J. DAPI: a DNA-specific fluorescent probe. Biotech Histochem. 1995 Sep;70(5): 220-33.

14 Beccia MR, Biver T, Pardini A, Spinelli J, Secco F, Venturini M, et al. The fluorophore 4',6-diamidino-2-phenylindole (DAPI) induces DNA folding in long double-stranded DNA. Chem Asian J. 2012 Aug;7(8):1803-10.

15 Matsuura M, Abe H, Tominaga T, Sakurai A, Murakami T, Kishi S, et al. A Novel Method of DAPI Staining for Differential Diagnosis of Renal Amyloidosis. J Med Invest. 2017 Aug; 64(3.4):217-21.4

16 Ollague W. Primary cutaneous amyloidosis. Int J Dermatol. 1987 Mar;26(2):135.

17 Breathnach SM. Amyloid and amyloidosis. J Am Acad Dermatol. 1988 Jan;18(1 Pt 1):1-16.

18 Westermark P, Araki S, Benson MD, Cohen AS, Frangione B, Masters CL, et al. Nomenclature of amyloid fibril proteins. Report from the meeting of the International Nomenclature Committee on Amyloidosis, August 8-9, 1998. Part 1. Amyloid. 1999 Mar;6(1):63-6.

19 Breathnach SM, Melrose SM, Bhogal B, de Beer FC, Black MM, Pepys MB. Ultrastructural localization of amyloid $\mathrm{P}$ component in primary localized cutaneous amyloidosis. Clin Exp Dermatol. 1983 Jul;8(4):355-62.

20 Chang YT, Tsai SF, Wang WJ, Hong CJ, Huang CY, Wong CK. A study of apolipoproteins E and A-I in cutaneous amyloids. Br J Dermatol. 2001 Sep;145(3):422-7.

21 Chapman JR, Liu A, Yi SS, Hernandez E, Ritorto MS, Jungbluth AA, et al. Proteomic analysis shows that the main constituent of subepidermal localised cutaneous amyloidosis is not galectin-7. Amyloid. 2021 Mar;28(1):35-41.

22 Yang CF, Lin SP, Chiang CP, Wu YH, H'ng WS, Chang CP, et al. Loss of GPNMB Causes Autosomal-Recessive Amyloidosis Cutis Dyschromica in Humans. Am J Hum Genet. 2018 Feb 1;102(2):219-32.
23 Brinster N, Calonje E In: Calonje E, Brenn T, Lazar A, McKee P, editors. Degenerative and metabolic diseases. McKee's Pathology of the Skin. China: Elsevier Saunders; 2012. Vol. 1. p. 520-89

24 Stokes G. An improved Congo red method for amyloid. Med Lab Sci. 1976 Jan;33(1):79-80.

25 Sari Aslani F, Kargar H, Safaei A, Jowkar F, Hosseini M, Sepaskhah M. Comparison of Immunostaining with Hematoxylin-Eosin and Special Stains in the Diagnosis of Cutaneous Macular Amyloidosis. Cureus. 2020 Apr 9; 12(4):e7606.

26 Maize JC, Ralston JS. Metabolic diseases of the skin. In: Elder DE, editor. Lever's Histopathology of the Skin. Philadelphia: Wolters Kluwer; 2015. p. 502-44.

27 Lin MW, Lee DD, Liu TT, Lin YF, Chen SY, Huang CC, et al. Novel IL31RA gene mutation and ancestral OSMR mutant allele in familial primary cutaneous amyloidosis. Eur J Hum Genet. 2010 Jan;18(1):26-32.

28 Arita K, South AP, Hans-Filho G, Sakuma TH, Lai-Cheong J, Clements S, et al. Oncostatin M receptor-beta mutations underlie familial primary localized cutaneous amyloidosis. Am J Hum Genet. 2008 Jan;82(1):73-80.

29 Wang J, Li Y, Xing L, Zhao M, Zhang S, Li Z, et al. Three novel mutations in GPNMB in two pedigrees with amyloidosis cutis dyschromica. Br J Dermatol. 2019 Dec;181(6):1327-9.

30 Ueo D, Utani A, Okubo Y, Yozaki M, Mine Y, Anan T, et al. Familial primary localized cutaneous amyloidosis in a Japanese family. J Dermatol Sci. 2016 Aug;83(2):162-4.

31 Lu P, Wu FF, Rong ZL, Fang C, Deng CC, Bin $\mathrm{LH}$, et al. Clinical and genetic features of Chinese patients with lichen and macular primary localized cutaneous amyloidosis. Clin Exp Dermatol. 2019 Jun;44(4):e110-7.

32 Gusel'nikova V, Antimonova O, Fedorova E, Shavlovsky M, Krutikov A, Mikhailova E, et al. Fluorescent characterization of amyloid deposits in the kidneys of mdx mice. Eur J Histochem. 2018 Apr 3;62(2):2870. 